\title{
EFEITO DE GRUPO GENÉTICO E HETEROSE SOBRE A IDADE E PESO À PUBERDADE E SOBRE O DESEMPENHO REPRODUTIVO DE NOVILHAS DE CORTE ${ }^{1}$
}

\author{
JOÃO RESTLE², VOLMIR ANTONIO POLLI ${ }^{3}$ e DILCEU BORGES DE SENNA ${ }^{4}$
}

\begin{abstract}
RESUMO - Foram avaliados os efeitos de grupo genético e heterose sobre a idade e peso à puberdade, bem como sobre o desempenho reprodutivo no primeiro período de reprodução de fêmeas de corte. Foram utilizadas 74 fêmeas nascidas em 1987 e 1988, das raças Charolês (C) e Nelore (N) e suas cruzas recíprocas $1 / 2 \mathrm{CN}$ e $1 / 2 \mathrm{NC}$. As fêmeas foram mantidas em pastagem cultivada durante o primeiro e segundo inverno e em campo nativo no resto do tempo. A heterose no peso vivo dos 7 aos 28 meses oscilou entre 12,8 e $14,6 \%$. Fêmeas cruzadas, $1 / 2 \mathrm{CN}$ e $1 / 2 \mathrm{NC}$, foram em média 89 dias mais precoces na idade à puberdade, resultando uma heterose de $-12,9 \%$. A idade média à puberdade foi de $623,754,568$ e 634 dias, respectivamente, para C, N, 1/2 CN e 1/2 NC, sendo os pesos médios correspondentes $352,299,323$ e $368 \mathrm{~kg}$. A heterose no peso à puberdade foi de $6,1 \%$, enquanto na percentagem de fêmeas que manifestaram cio até os 18, 24 e 28 meses de idade foi, respectivamente, de $76,9,67,6$ e 17,6\%. A percentagem de prenhez no final do período reprodutivo, dos 25 aos 28 meses, foi de $94,4,53,4,99,4$ e $97,5 \%$, respectivamente para C, N, 1/2 CN e 1/2 NC. Fêmeas mais pesadas ao desmame e aos 12 meses foram mais precoces na manifestação da puberdade, sendo os coeficientes de correlação iguais a $-0,39$ e $-0,56$, respectivamente.
\end{abstract}

Termos para indexação: Charolês, cruzamento, Nelore, reprodução.

\section{BREED AND HETEROSIS EFFECT ON AGE AND WEIGHT AT PUBERTY AND REPRODUCTIVE PERFORMANCE OF BEEF HEIFERS}

\begin{abstract}
The effect of breed and heterosis on age and weight at puberty and reproductive performance at the first breeding season of beef heifers were evaluated. Seventy four Charolais (C), Nellore (N), 1/2 CN and $1 / 2$ NC females, born in 1987 and 1988, were used. The females were kept on cultivated pasture during the first and second winter, and on native pasture the rest of the time. Heterosis for live weight at the different ages, ranged from 12.8 to $14.6 \%$. Crossbred heifers, $1 / 2 \mathrm{CN}$ and $1 / 2 \mathrm{NC}$, reached puberty 89 days earlier than straightbreds, resulting a heterosis of $-12.9 \%$. Average age at puberty was $623,754,568$ and 634 days, respectively, for C, N, 1/2 CN and 1/2 NC. Average live weight at puberty was $352,299,323$ and $368 \mathrm{~kg}$, respectively. Heterosis for percentage of heifers showing heat until 18, 24 and 28 months of age was 76.9, 67.6 and $17.6 \%$, respectively. The percentage of heifers pregnant at the end of the first breeding season, 25 to 28 months, was $94.4,53.4,99.4$ and $97.5 \%$, respectively, for $\mathrm{C}, \mathrm{N}, 1 / 2 \mathrm{CN}$ and $1 / 2 \mathrm{NC}$. Heavier heifers at weaning and at 12 months reached puberty earlier, being the correlation coeficients of -.39 and -.56 , respectively.
\end{abstract}

Index terms: Charolais, crossbreeding, Nellore, reproduction.

${ }^{1}$ Aceito para publicação em 7 de julho de 1998.

${ }^{2}$ Eng. Agr., Ph.D., Prof. Titular, Dep. de Zootecnia, Universidade Federal de Santa Maria (UFSM), Campus Camobi, CEP 97119-900 Santa Maria, RS. Bolsista do CNPq. E-mail: jorestle@creta.ccr.ufsm.br

${ }^{3}$ Eng. Agr., M.Sc., Dep. de Zootecnia, UFSM.

${ }^{4}$ Zoot., Dep. de Zootecnia, UFSM.

\section{INTRODUÇÃO}

O início da atividade reprodutiva em fêmeas de corte tem grande influência no desempenho do rebanho de cria. No Rio Grande do Sul, a maioria das fêmeas atinge a puberdade com idade avançada, sendo acasaladas aos três anos e produzindo a sua primeira cria aos quatro anos. O longo período 
em que as fêmeas são mantidas na propriedade sem produzir eleva os custos de produção de bezerros, bem como atrasa o processo de seleção genética do rebanho.

A idade à puberdade, que em fêmeas tem sido definida como a idade da manifestação do primeiro cio, é influenciada por fatores ambientais, principalmente o nível de alimentação, e por fatores genéticos (Wiltbank et al., 1966).

$\mathrm{O}$ efeito da raça na idade à puberdade tem sido demonstrado por vários autores (Wiltbank et al., 1966; Preston \& Willis, 1970; Gregory et al., 1991). Por sua vez, os cruzamentos de reprodutores de diferentes raças dão origem a fêmeas que atingem a puberdade com menos idade do que as das raças puras (Wiltbank et al., 1966; Laster et al., 1976; Martin et al., 1992). Stewart et al. (1980) relatam uma heterose de $-15,4 \%$ para idade à puberdade.

Idade à puberdade em fêmeas é uma característica importante em gado de corte à medida que o sistema de produção se torna mais intensivo e competitivo. No entanto, são poucas as informações sobre os efeitos da raça e da heterose na idade e peso à puberdade em fêmeas de corte.

O presente experimento teve como objetivos avaliar o desenvolvimento ponderal, a idade e peso à puberdade, bem como a taxa de prenhez, no primeiro acasalamento, de fêmeas das raças Charolês, Nelore e suas cruzas recíprocas.

\section{MATERIAL E MÉTODOS}

O experimento foi desenvolvido entre dezembro de 1986 e abril de 1991, nas dependências do Departamento de Zootecnia da Universidade Federal de Santa Maria, RS.

O clima da região, segundo a classificação de Köppen é subtropical - cfa (Moreno, 1961). A temperatura média anual varia de 14,3 a $25,2^{\circ} \mathrm{C}$, com médias das máximas temperaturas de $29,9^{\circ} \mathrm{C}$ no mês de janeiro, e das mínimas de $9,7^{\circ} \mathrm{C}$ no mês de agosto, com possibilidade de ocorrência de geadas de abril a outubro. A média anual de umidade relativa do ar é de $73 \%$ e a precipitação de $1.650,9 \mathrm{~mm}$ (Rio Grande do Sul, 1979).

Foram utilizadas 74 fêmeas, nascidas no período de parição de setembro a dezembro de 1987 e 1988, das raças Charolês $(\mathrm{C})$ e Nelore $(\mathrm{N})$ puras e cruzas recíprocas entre elas, $1 / 2 \mathrm{CN}$ e $1 / 2 \mathrm{NC}$. As fêmeas foram geradas por vacas puras $\mathrm{C}$ e $\mathrm{N}$, inseminadas com o sêmen de 17 touros $\mathrm{C}$ e $\mathrm{N}$; e também submetidas ao repasse com oito touros, quatro por ano, no terço final do período de reprodução, cuja duração foi de 90 dias.

As fêmeas foram mantidas em regime de campo nativo com suas mães até o desmame, realizado com a idade média de sete meses. Dos sete aos 12 e dos 20 aos 24 meses permaneceram em pastagem cultivada de inverno composta de aveia (Avena strigosa) mais azevém (Lolium multiflorum), e no restante do tempo permaneceram em campo nativo. A lotação na pastagem cultivada foi de 2,2 unidades animal/ha no primeiro e segundo inverno. No campo nativo, a lotação foi de uma unidade animal/ha. Durante o período experimental os animais receberam sal + minerais nos respectivos pastos. As vacinações seguiram o calendário da Secretaria da Agricultura, RS. O controle de endo e ectoparasitas foi realizado com produtos específicos e de acordo com o grau de infestação.

Os dados coletados foram os pesos até os 28 meses de idade, idade e peso à puberdade e percentagem de prenhez no primeiro período de reprodução. Os animais foram pesados ao nascer, desmame e a cada 28 dias até completarem 28 meses de idade.

A idade à puberdade foi considerada como sendo a idade ao primeiro cio. Nas novilhas nascidas de setembro a dezembro de 1987, ela foi obtida a partir de julho de 1988 até o final do entouramento (fevereiro de 1990), e nas nascidas em 1988, esse dado foi obtido a partir de julho de 1989 até o final do entouramento (fevereiro de 1991). A observação em ambos os lotes foi feita percorrendo-se os pastos diariamente, com o auxílio de rufiões com buçal marcador.

O período destinado à primeira reprodução das novilhas nos dois anos teve a duração de 90 dias, com início no dia 1o de dezembro. Nos primeiros 23 dias do período reprodutivo foi utilizada a prática de inseminação artificial, e nos 67 dias restantes utilizaram-se touros com buçal marcador. As novilhas $\mathrm{C} \mathrm{e} 1 / 2 \mathrm{NC}$ foram inseminadas com sêmen ou acasaladas com touros $\mathrm{C}$, e as $\mathrm{N}$ e $1 / 2 \mathrm{CN}$ com N.

$\mathrm{O}$ delineamento experimental foi inteiramente casualizado em um arranjo fatorial $4 \times 2$, efetuando-se as análises, seguindo o modelo abaixo:

Yijklmn $=\mathrm{M}+\mathrm{SAi}+\mathrm{GGj}(\mathrm{SAi})+\mathrm{EAk}+$ $(\mathrm{EA} * \mathrm{GG}(\mathrm{SAi})) \mathrm{kj}+(\mathrm{SA} * \mathrm{EA}) \mathrm{ik}+\mathrm{OPl}+\mathrm{IMm}+$ Eijklmn onde:

Yijklmn = variáveis dependentes;

$\mathrm{M}=$ média de todas as observações;

$\mathrm{SAi}=$ efeito do sistema de acasalamento de índice $i$, sendo $\boldsymbol{i}=1$ (puras) e 2 (cruzadas); 
GGj $(\mathrm{SAi})=$ efeito do grupo genético de índice $\boldsymbol{j}$, sendo $\boldsymbol{j}=1$ (Charolês), 2 (Nelore), 3 (1/2 CN), e 4 (1/2 NC), dentro do sistema de acasalamento $\boldsymbol{i}$;

$\mathrm{EAk}=$ efeito do ano de nascimento de índice $\boldsymbol{k}$, sendo $\boldsymbol{k}=1$ (novilhas nascidas em 1987) e 2 (novilhas nascidas em 1988);

$(\mathrm{EA} * \mathrm{GG}(\mathrm{SAi})) \mathrm{kj}$ = interação entre grupo genético de índice $\boldsymbol{j}$, dentro do sistema de acasalamento $\boldsymbol{i}$, com o efeito do ano de nascimento de índice $\boldsymbol{k}$;

$(\mathrm{SA} * \mathrm{EA}) \mathrm{ik}=$ interação entre o sistema de acasalamento de índice $\boldsymbol{i}$, e efeito do ano de nascimento de índice $\boldsymbol{k}$;

OPl = covariável ordem de parição de índice $l$;

IMm = covariável idade da mãe de ordem $\boldsymbol{m}$; e

Eijklmn = erro aleatório assumindo distribuição normal com média igual a zero e variância $\boldsymbol{\sigma}^{-2}$.

Os dados foram analisados pelo método dos quadrados mínimos (SAS Institute, 1985).

As covariáveis ordem de parição e idade da mãe foram incluídas no modelo para análise das seguintes variáveis: peso ao desmame, peso aos 18, 24 e 28 meses, idade e peso à puberdade, e diagnóstico de gestação.

\section{RESULTADOS E DISCUSSÃO}

Os resultados referentes aos pesos nas diferentes idades são apresentados na Tabela 1. A média do peso das novilhas $\mathrm{F}_{1}$ foi superior à média das puras em todas as idades avaliadas, com a heterose oscilando entre 12,8 e 14,6\%. A heterose observada foi superior àquela registrada por Restle et al. (1995) em novilhos aos 24 meses de idade $(9,7 \%)$ envolvendo os mesmos grupos genéticos, porém em melhores condições de alimentação.

Observa-se que nas novilhas puras as $\mathrm{C}$ foram mais pesadas que as $\mathrm{N}$ em todos os períodos avaliados, com a diferença chegando a $30 \%$ aos 12 meses de idade. $\mathrm{O}$ maior ganho de peso das fêmeas $\mathrm{C}$ deve-se principalmente à seleção intensa, para essa característica, a que foi submetida a raça durante sua formação. Apesar de os pesos das $\mathrm{N}$ terem sido menores aos dos outros grupos genéticos, esses foram superiores aos citados por Alencar et al. (1987) e aos obtidos no Estado de São Paulo. Comparando as fêmeas $F_{1}$, verifica-se que os pesos foram similares $(\mathrm{P}>0,05)$ entre as $1 / 2 \mathrm{CN}$ e as $1 / 2 \mathrm{NC}$ em todas as idades avaliadas.
Na Tabela 2 são apresentados os resultados referentes ao peso médio e idade média à puberdade. Não foi constatada diferença significativa $(\mathrm{P}>0,05)$ entre as fêmeas puras e as cruzadas em relação ao peso à puberdade, sendo a heterose de apenas $6,1 \%$. Apesar de a diferença de $18 \mathrm{~kg}$ a favor das cruzadas não ter sido significativa, provavelmente devido ao pequeno número de unidades experimentais, ela foi superior aos $6,8 \mathrm{~kg}$ encontrados por Gregory et al. (1978) em trabalho envolvendo várias raças européias, e aos $11 \mathrm{~kg}$ obtidos por Stewart et al. (1980).

$\mathrm{O}$ peso à puberdade das $\mathrm{C}$ foi $53 \mathrm{~kg}$ superior $(\mathrm{P}>0,05)$ ao das $\mathrm{N}$, e entre as cruzas a diferença foi de $45 \mathrm{~kg}$ em favor das $1 / 2 \mathrm{NC}(\mathrm{P}>0,05)$. Os pesos à puberdade das fêmeas $\mathrm{N}$ e das cruzadas foram superiores aos relatados por Alencar et al. (1987), que obtiveram em fêmeas $\mathrm{N}$ e Canchim pesos à puberdade de 282 e $293 \mathrm{~kg}$, respectivamente. O peso à puberdade das fêmeas $\mathrm{C}$ foi similar ao relatado por Martin et al. (1992).

Quanto à idade à puberdade constata-se que as cruzadas foram na média 89 dias mais precoces na manifestação do primeiro cio, mostrando uma heterose de $-12,9 \%$. Essa constatação está de acordo com Wiltbank et al. (1966), Laster et al. (1976), Stewart et al. (1980) e Martin et al. (1992), ao observarem que as fêmeas cruzadas são mais precoces na manifestação do primeiro cio que as de raças puras. Wiltbank et al. (1966) mostram heterose de $-9,5$ e $-9,8 \%$ em cruzamentos envolvendo as raças A. Angus, Hereford e Shorthorn submetidas a um baixo ou alto nível nutricional, respectivamente. Stewart et al. (1980) trabalhando com as raças A. Angus, Brahman, Holandês, Jersey e Hereford relatam heterose média de $-15 \%$.

As novilhas $\mathrm{C}$ foram mais precoces $(\mathrm{P}<0,05)$ do que as $\mathrm{N}$ na manifestação do primeiro cio. Entre as cruzas não foi verificada diferença significativa. Alencar et al. (1987) relatam idade à puberdade superior às aqui obtidas, sendo 730 dias com fêmeas Canchin e 772 dias com N. Por sua vez, com fêmeas C, Gregory et al. (1991) citam idade à puberdade bem inferior, de 369 dias.

Comparando os dados deste experimento com os obtidos em meio ambiente mais favorável e citados por Gregory et al. (1991), verifica-se que nos dois 
TABELA 1. Peso médio (kg) ajustado e erro padrão das novilhas aos 7, 12, 18, 24 e 28 meses de idade, de acordo com o sistema de acasalamento e grupo genético ${ }^{1}$.

\begin{tabular}{|c|c|c|c|c|c|c|}
\hline \multirow{2}{*}{$\begin{array}{l}\text { Grupo } \\
\text { genético }\end{array}$} & \multirow{2}{*}{$\begin{array}{l}\text { Número de } \\
\text { animais }\end{array}$} & \multicolumn{5}{|c|}{ Idade (meses) } \\
\hline & & 7 & 12 & 18 & 24 & 28 \\
\hline Novilhas puras & 38 & & & & & \\
\hline Charolês (C) & 22 & $178 \pm 7 a$ & $233 \pm 6 a$ & $292 \pm 6 a$ & $357 \pm 8 \mathrm{a}$ & $414 \pm 8 \mathrm{a}$ \\
\hline Nelore (N) & 16 & $146 \pm 7 b$ & $179 \pm 6 b$ & $244 \pm 6 b$ & $300 \pm 8 b$ & $343 \pm 8 b$ \\
\hline Média & & $162 \pm 5 \mathrm{~B}$ & $206 \pm 4 \mathrm{~B}$ & $268 \pm 4 \mathrm{~B}$ & $329 \pm 6 \mathrm{~B}$ & $378 \pm 6 \mathrm{~B}$ \\
\hline Novilhas cruzadas & 36 & & & & & \\
\hline $1 / 2 \mathrm{CN}$ & 14 & $184 \pm 8 \mathrm{a}$ & $236 \pm 7 a$ & $308 \pm 7 a$ & $377 \pm 9 a$ & $435 \pm 9 a$ \\
\hline $1 / 2 \mathrm{NC}$ & 22 & $181 \pm 6 a$ & $235 \pm 6 a$ & $306 \pm 6 a$ & $365 \pm 8 a$ & $423 \pm 7 a$ \\
\hline Média & & $183 \pm 5 \mathrm{~A}$ & $235 \pm 4 \mathrm{~A}$ & $307 \pm 4 \mathrm{~A}$ & $371 \pm 6 \mathrm{~A}$ & $429 \pm 6 \mathrm{~A}$ \\
\hline Heterose $(\%)$ & & 13,0 & 14,1 & 14,6 & 12,8 & 13,5 \\
\hline
\end{tabular}

${ }^{1} \mathrm{a}, \mathrm{b}(\mathrm{P}<0,05)$ na mesma coluna para Charolês vs Nelore e $1 / 2 \mathrm{CN}$ vs $1 \frac{1}{2} \mathrm{NC} ; \mathrm{A}, \mathrm{B}(\mathrm{P}<0,05)$ na mesma coluna para puras vs cruzadas.

TABELA 2. Média e erro padrão para peso $(\mathrm{kg})$ e idade média (dias) ajustados das novilhas à puberdade, de acordo com o sistema de acasalamento e grupo genético ${ }^{1}$.

\begin{tabular}{lccc}
\hline $\begin{array}{l}\text { Grupo } \\
\text { genético }\end{array}$ & $\begin{array}{c}\text { Número de } \\
\text { animais }\end{array}$ & Peso & Idade \\
\hline Novilhas puras & 38 & $352 \pm 15 \mathrm{a}$ & $623 \pm 34 \mathrm{~b}$ \\
Charolês (C) & 22 & $299 \pm 20 \mathrm{a}$ & $754 \pm 36 \mathrm{a}$ \\
Nelore (N) & 16 & $326 \pm 12 \mathrm{~A}$ & $689 \pm 25 \mathrm{~A}$ \\
Média das puras & & & \\
Novilhas cruzadas & 36 & $323 \pm 17 \mathrm{a}$ & $568 \pm 39 \mathrm{a}$ \\
$1 / 2 \mathrm{CN}$ & 14 & $368 \pm 14 \mathrm{a}$ & $634 \pm 31 \mathrm{a}$ \\
$1 / 2 \mathrm{NC}$ & 22 & $346 \pm 11 \mathrm{~A}$ & $600 \pm 24 \mathrm{~B}$ \\
Média cruzadas & & 6,1 & $-12,9$ \\
Heterose (\%) & & & \\
\hline
\end{tabular}

${ }^{1} \mathrm{a}, \mathrm{b}(\mathrm{P}<0,05)$ na mesma coluna para Charolês vs Nelore e $1 / 2 \mathrm{CN}$ vs $1 / 2 \mathrm{NC} ; \mathrm{A}, \mathrm{B}(\mathrm{P}<0,05)$ na mesma coluna para puras vs cruzadas.

casos o peso à puberdade das fêmeas $\mathrm{C}$ foi similar, no entanto a idade foi muito diferente, ou seja, 626 dias no presente trabalho versus os 391 dias citados por aqueles autores. Tal constatação mostra que para manifestar a puberdade é fundamental que a fêmea atinja um determinado grau de desenvolvimento, e que idade à puberdade é principalmente uma consequiência da velocidade de ganho de peso, que por sua vez está condicionando ao meio ambiente.
A correlação entre idade e peso à puberdade mostrou um coeficiente de $0,70(\mathrm{P}<0,01)$, indicando que as novilhas mais tardias à puberdade também foram mais pesadas. $O$ coeficiente de correlação encontrado é intermediário aos 0,57 obtido por Arije \& Wiltbank (1971) em novilhas Hereford, e aos 0,90 relatados por Laster et al. (1976).

O peso ao desmame praticamente não teve associação com o peso à puberdade $(\mathrm{r}=0,18$; $\mathrm{P}>0,05)$, mas quando correlacionado com a idade à puberdade esta foi negativa $(\mathrm{r}=-0,39 ; \mathrm{P}<0,01)$, mostrando que novilhas mais pesadas ao desmame são mais precoces. Assim, peso ao desmame se torna cada vez mais importante à medida que a produção de gado de corte é intensificada e mais competitiva. Segundo Patterson et al. (1992) o crescimento pré-desmame exerce maior influência na puberdade de novilhas de corte do que taxas de crescimento pós-desmame. Arije e Wiltbank (1971) observaram que novilhas que faziam melhores ganhos pós-desmame foram mais pesadas à puberdade, mas não necessariamente mais jovens. Concordando com o que já foi afirmado acima, Plasse et al. (1968) encontraram correlações entre peso ao desmame (285 dias) e idade à puberdade de $-0,46$ e $-0,41$ na raça Brahman e cruzas, respectivamente. O peso aos 12 meses também foi 
correlacionado negativamente $(r=-0,56$; $\mathrm{P}<0,01)$ com idade à puberdade. $\mathrm{O}$ mesmo foi observado por Smith et al. (1976).

$\mathrm{Na}$ Tabela 3 constam os valores referentes à percentagem de fêmeas em cio nas diferentes idades. Comparando a média das fêmeas puras com a média das cruzadas, verifica-se que em todas as idades avaliadas as cruzadas apresentaram maiores percentagens de cio. A diferença, embora tenha decrescido com o avanço da idade, foi significativa $(\mathrm{P}<0,05)$ em todas as fases. Tal diferença é melhor expressa pela heterose de 76,9, 67,6 e 17,6\% aos 18,24 e 28 meses, respectivamente. A diminuição na heterose verificada com o avanço da idade é igualmente salientada por Martin et al. (1992).

Laster et al. (1976) trabalhando com as raças A. Angus e Hereford obtiveram níveis de heterose, na percentagem de fêmeas que atingiram a puberdade em vários intervalos de idade, inferiores aos verificados no presente experimento. Esses maiores níveis de heterose observados são em parte decorrentes das raças utilizadas nos dois cruzamentos, já que existe maior diversidade genética envolvida quando se cruzam raças zebuínas com européias do que quando raças européias são cruzadas entre si (Koger, 1976).

Verificou-se que aos 12 meses nenhuma das fêmeas puras havia manifestado cio contra uma pequena percentagem das cruzadas. A percentagem de fêmeas em cio aos 18 meses é particularmente importante se for considerado um sistema mais intensivo de produção em que o primeiro período reprodutivo é realizado dos 15 aos 18 meses de idade. Verificou-se que nesse caso apenas o grupo genético $\mathrm{CN}$ apresentou uma percentagem considerável de fêmeas em cio. A maior precocidade foi o resultado do maior peso apresentado por essas fêmeas no desmame aos sete meses e nas demais idades. Até o final do primeiro período reprodutivo todas as fêmeas $\mathrm{C}, \mathrm{CN}$ e $\mathrm{NC}$ haviam manifestado cio, já nas N 30\% não haviam manifestado.

A percentagem de novilhas prenhes no final do período reprodutivo consta na Tabela 4. A percentagem de prenhez das fêmeas cruzadas foi superior ( $\mathrm{P}<0,05)$ ao das puras, sendo a heterose de 33,3\%. Steffan et al. (1985) acasalando fêmeas a partir dos 15 meses de idade também observaram menor percentagem de prenhez nas fêmeas puras em relação às cruzadas, o que também foi constatado por Laster et al. (1976), que obtiveram $15,9 \%$ a mais em novilhas $F_{1}$ do que nas puras.

Entre os grupos genéticos a menor percentagem de prenhez foi observada nas $\mathrm{N}$, o que em parte deveu-se à idade avançada com que essas fêmeas atingiram a puberdade. A correlação entre idade à puberdade e percentagem de prenhez foi de $-0,35(\mathrm{P}<0,01)$, constatação similar foi feita por Laster et al. (1979) que obtiveram uma correlação de $-0,42$ entre as duas variáveis.

TABELA 3. Média ajustada e erro padrão para percentagem de novilhas que mostraram cio até os 12, 18, 24 e 28 meses de idade, de acordo com o sistema de acasalamento e grupo genético ${ }^{1}$.

\begin{tabular}{|c|c|c|c|c|c|}
\hline \multirow{2}{*}{$\begin{array}{l}\text { Grupo } \\
\text { genético }\end{array}$} & \multirow{2}{*}{$\begin{array}{l}\text { Número de } \\
\text { animais }\end{array}$} & \multicolumn{4}{|c|}{ Idade (meses) } \\
\hline & & 12 & 18 & 24 & 28 \\
\hline Novilhas puras & 38 & & & & \\
\hline Charolês (C) & 22 & 0 & $40,6 \pm 11,3 \mathrm{a}$ & $56,5 \pm 1,0 \mathrm{a}$ & $100,0 \pm 4,8 \mathrm{a}$ \\
\hline Nelore (N) & 16 & 0 & $16,7 \pm 11,5 \mathrm{a}$ & $25,0 \pm 11,2 b$ & $70,0 \pm 4,9 \mathrm{~b}$ \\
\hline Média & & 0 & $28,6 \pm 8,0 \mathrm{~B}$ & $40,7 \pm 7,9 \mathrm{~B}$ & $85,0 \pm 3,4 \mathrm{~B}$ \\
\hline Novilhas cruzadas & 36 & & & & \\
\hline $1 / 2 \mathrm{CN}$ & 14 & $11,1 \pm 5,4$ & $67,8 \pm 12,4 a$ & $78,9 \pm 12,1 \mathrm{a}$ & $100,0 \pm 5,3 \mathrm{a}$ \\
\hline $1 / 2 \mathrm{NC}$ & 22 & $3,3 \pm 4,4$ & $33,3 \pm 10,2 b$ & $57,6 \pm 9,9 \mathrm{a}$ & $100,0 \pm 4,4 \mathrm{a}$ \\
\hline Média & & $7,2 \pm 3,5$ & $50,6 \pm 8,0 \mathrm{~A}$ & $68,2 \pm 7,8 \mathrm{~A}$ & $100,0 \pm 3,4 \mathrm{~A}$ \\
\hline Heterose $(\%)$ & & - & 76,9 & 67,6 & 17,6 \\
\hline
\end{tabular}

${ }^{1}$ a, b $(\mathrm{P}<0,05)$ na mesma coluna para Charolês vs Nelore e $1 / 2 \mathrm{CN}$ vs $1 \frac{1}{2} \mathrm{NC} ; \mathrm{A}, \mathrm{B}(\mathrm{P}<0,05)$ na mesma coluna para puros vs cruzadas. 
TABELA 4. Média ajustada e erro padrão para percentagem de prenhez de novilhas ao final do primeiro período reprodutivo, de acordo com o sistema de acasalamento e grupo genético ${ }^{1}$.

\begin{tabular}{lcc}
\hline Grupo genético & $\begin{array}{c}\text { Número de } \\
\text { animais }\end{array}$ & $\begin{array}{c}\text { Percentagem de } \\
\text { prenhez }\end{array}$ \\
\hline Novilhas puras & 38 & \\
Charolês (C) & 22 & $94,4 \pm 8,1 \mathrm{a}$ \\
Nelore (N) & 16 & $53,4 \pm 8,3 \mathrm{~b}$ \\
Média & & $73,9 \pm 5,7 \mathrm{~B}$ \\
& & \\
Novilhas cruzadas & 36 & $99,4 \pm 9,1 \mathrm{a}$ \\
1/2 CN & 14 & $97,5 \pm 7,4 \mathrm{a}$ \\
1/2 NC & 22 & $98,5 \pm 5,8 \mathrm{~A}$ \\
Média & & 33,3 \\
Heterose $(\%)$ & & \\
\hline 1 a, b (P < 0,05) na mesma coluna para Charolês vs Nelore $\mathrm{e}^{1 / 2} \mathrm{CN}$ vs $1 / 2 \mathrm{NC} ;$ \\
A, B (P $<0,05)$ na mesma coluna para puras vs cruzadas.
\end{tabular}

\section{CONCLUSÕES}

1. São expressivos os níveis de heterose no desenvolvimento ponderal, idade à puberdade e desempenho reprodutivo de novilhas.

2. Fêmeas Charolês são mais precoces e mais pesadas na manifestação do primeiro cio e apresentam maior taxa de prenhez no primeiro acasalamento do que fêmeas Nelore.

3. Fêmeas mais pesadas ao desmame são mais precoces na manifestação da atividade sexual.

\section{REFERÊNCIAS}

ALENCAR, M.M. de; COSTA, J.L. da; CORRÊA, L. de A. Desempenho reprodutivo de fêmeas das raças Canchim e Nelore. 1. Desenvolvimento e puberdade. Pesquisa Agropecuária Brasileira, Brasília, v.22, n.7, p.753-758, jul. 1987.

ARIJE, F.G.; WILTBANK, J.N. Age and weight at puberty in Hereford heifers. Journal of Animal Science, Champaign, v.33, n.2, p.401-406, Aug. 1971.

GREGORY, K.E.; LASTER, D.B.; CUNDIFF, L.V.; SMITH, G.M.; KOCH, R.M. Heterosis and breed maternal and transmitted effects in beef catle.
II. Growth rate and puberty in females. Journal of Animal Science, Champaign, v.47, n.5, p.1042-1053, Nov. 1978.

GREGORY, K.E.; LUNSTRA, D.D.; CUNDIFF, L.V.; $\mathrm{KOCH}, \mathrm{R} . \mathrm{M}$. Breed effects and heterosis in advanced generations of composite populations for puberty and scrotal traits of beef cattle. Journal of Animal Science, Champaign, v.69, n.7, p.2795-2807, July 1991.

KOGER, M. Resumen y conclusiones. In: KOGER, M.; CUNHA, T.J.; WARNICK. A.C. (Eds.). Cruzamientos en ganado vacuno de carne. Montevideo: Hemisfério Sur, 1976. Cap.43, p.536-552.

LASTER, D.B.; SMITH, G.M.; CUNDIFF, L.V.; GREGORY, K.E. Characterization of biological types of cattle (cicle II). II. Postweaning growth and puberty of heifers. Journal of Animal Science, Champaign, v.48, n.3, p.500-508, Mar. 1979.

LASTER, D.B.; SMITH, G.M.; GREGORY, K.E. Characterization of biological types of cattle. IV. Postweaning growth and puberty of heifers. Journal of Animal Science, Champaign, v.43, n.1, p.63-70, Jan. 1976.

MARTIN, L.C.; BRINKS, J.S.; BOURDON, R.M.; CUNDIFF, L.V. Genetic effects on beef heifer puberty and subsequent reproduction. Journal of Animal Science, Champaign, v.70, n.12, p.4006-4017, Dec. 1992.

MORENO, J.A. Clima do Rio Grande do Sul. Porto Alegre: Secretaria da Agricultura, 1961. 41p.

PATTERSON, D.J.; CORAH, L.R.; BRETHOUR, J.R. Evaluation of reprodutive traits in Bos tauros and Bos indicus crossbred heifers: relationship of age at puberty to length of the postpartum interval to estrus. Journal of Animal Science, Champaign, v.70, n.7, p.1994-1999, July 1992.

PLASSE, D.; WARNICK, A.C.; KOGER, M. Reprodutive behavior of Bos indicus females in a subtropical environment. I. Puberty and ovulation frequency in Brahman and Brahman x British heifers. Journal of Animal Science, Champaign, v.27, n.1, p.94-100, Jan. 1968.

PRESTON, T.R.; WILLIS, M.B. Intensive beef production. Oxford: Pergamon Press, 1970. 544p. 
RESTLE, J.; FELTEN, H.G.; VAZ, F.N. Efeito de raça e heterose para desempenho em confinamento de novilhos de corte. Revista Argentina de Produción Animal, Mar del Plata, v.15, n.3/4, p.852-854, nov. 1995.

RIO GRANDE DO SUL. Secretaria da Agricultura. Observações meteorológicas no Estado de Rio Grande do Sul. Porto Alegre, 1979. 270p. (Boletim técnico, 3).

SAS INSTITUTE. Statistical analysis system: user's guide. Version 6. 4.ed. Cary, NC, 1985. 956p.

SMITH, G.M.; FITZHUGH JUNIOR, H.A.; CUNDIFF, L.V.; CARTWRIGHT, T.C.; GREGORY, K.E. A genetic analysis of maturing patterns in straightbred and crossbred Hereford, Angus and Shorthorn cattle. Journal of Animal Science, Champaign, v.43, n.2, p.389-396, Feb.1976.
STEFFAN, C.A.; KRESS, D.D.; DOORNBOS, D.E. Performance of crosses among Hereford, Angus and Simental cattle with different levels of Simmental breeding. III. Heifer postweaning growth and early reproductive traits. Journal of Animal Science, Champaign, v.61, n.5, p.1111-1120, May 1985.

STEWART, T.S.; LONG, C.R.; CARTWRIGHT, T.C. Characterization of cattle of a five-breed diallel. III. Puberty in bulls and heifers. Journal of Animal Science, Champaign, v.50, n.5, p.808-820, May 1980.

WILTBANK, J.N.; GREGORY, K.E.; SWIGER, L.A.; INGALLS, J.E. Effects of heterosis on age and weight at puberty in beef heifers. Journal of Animal Science, Champaign, v.25, n.3, p.744-751, Aug. 1966. 